\title{
N-Type Calcium Channels Control GABAergic Transmission in Brain Areas related to Fear and Anxiety
}

Maxwell Blazon, Brianna LaCarubba, Alexandra Bunda, Natalie Czepiel, Shayna Mallat, Laura Londrigan, Arturo Andrade *

Department of Biological Sciences, University of New Hampshire. 46 College Road, 245 Rudman Hall. Durham, NH, USA; E-Mails: blazon94@gmail.com; lacarubb@gmail.com; lexi.bunda@gmail.com; njc1037@wildcats.unh.edu; $\quad$ I1043@wildcats.unh.edu;

Arturo.Andrade@unh.edu

* Correspondence: Arturo Andrade; E-Mail: Arturo.Andrade@unh.edu

Academic Editor: Vito Di Maio

Special Issue: Synapses and Synaptic Function

OBM Neurobiology

2021, volume 5 , issue 1

doi:10.21926/obm.neurobiol.2101083
Received: September 13, 2020

Accepted: January 03, 2021

Published: January 12, 2021

\begin{abstract}
$\mathrm{N}$-type (Cav2.2) calcium channels are key for action potential-evoked transmitter release in the peripheral and central nervous system. Previous studies have highlighted the functional relevance of $\mathrm{N}$-type calcium channels at both the peripheral and central level. In the periphery, $\mathrm{N}$-type calcium channels regulate nociceptive and sympathetic responses. At the central level, $\mathrm{N}$-type calcium channels have been linked to aggression, hyperlocomotion, and anxiety. Among the areas of the brain that are involved in anxiety are the basolateral amygdala, medial prefrontal cortex, and ventral hippocampus. These three areas share similar characteristics in their neuronal circuitry, where pyramidal projection neurons are under the inhibitory control of a wide array of interneurons including those that express the peptide cholecystokinin. This type of interneuron is well-known to rely on $\mathrm{N}$-type calcium channels to release GABA in the hippocampus, however, whether these channels control GABA release from cholecystokininexpressing interneurons in the basolateral amygdala and medial prefrontal cortex is not known. Here, using mouse models to genetically label cholecystokinin-expressing
\end{abstract}

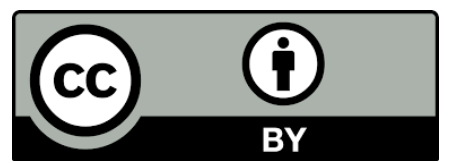

(C) 2021 by the author. This is an open access article distributed under the conditions of the Creative Commons by Attribution License, which permits unrestricted use, distribution, and reproduction in any medium or format, provided the original work is correctly cited. 
interneurons and electrophysiology, we found that in the basolateral amygdala, N-type calcium channels control $\sim 50 \%$ of GABA release from these neurons onto pyramidal cells. By contrast, in the medial prefrontal cortex $\mathrm{N}$-type calcium channels are functionally absent in synapses of cholecystokinin-expressing interneurons, but control $40 \%$ of GABA release from other types of interneurons. Our findings provide insights into the precise localization of $\mathrm{N}$ type calcium channels in interneurons of brain areas related to anxiety.

\section{Keywords}

N-type calcium channels; Cav2.2; medial prefrontal cortex; cholecystokinin-expressing interneurons; basolateral amygdala; hippocampus; GABA release, anxiety

\section{Introduction}

$\mathrm{N}$-type (Cav2.2) calcium channels open in response to action potentials, thereby contributing to neurotransmitter release. $\mathrm{N}$-type calcium channels, together with $\mathrm{P} / \mathrm{Q}$-type (Cav2.1) and R-type (Cav2.3) calcium channels, mediate neurotransmission in peripheral and central synapses [1-3]. In the periphery, $\mathrm{N}$-type calcium channels are essential for the release of neurotransmitters from nociceptors in neurons of the dorsal horn of the spinal cord [4]. Furthermore, N-type calcium channels control the release of adrenaline from postganglionic sympathetic neurons [5-8]. In the central nervous system, N-type calcium channels control transmitter release in specific areas. For example, dopaminergic neurons of the substantia nigra and ventral tegmental area heavily rely on $\mathrm{N}$-type calcium channels to release dopamine [9-11]. In the hippocampus (HPC), interneurons that express the peptide cholecystokinin ( $\mathrm{CCK}^{+} \mathrm{INs}$ ) fully rely on $\mathrm{N}$-type calcium channels to release GABA [12-16]. In terminals of pyramidal projection neurons (PNs), N-type calcium channels work together with $\mathrm{P} / \mathrm{Q}$ - and R-type calcium channels to regulate glutamate release [17-19]. Thus, disruption in $\mathrm{N}$ type calcium channel activity leads to alterations of peripheral and central nervous system functions.

Behavioral studies in the Cav2.2-null mice highlight the functional role of $\mathrm{N}$-type calcium channels in regulating processes related to the central nervous system. For example, Cav2.2-null mice exhibit hyperlocomotion, enhanced aggression, enhanced response to apomorphine, reduced exploratory behavior, and increased freezing during startle [20-22]. The latter two observations implicate Ntype calcium channels in anxiety-related behaviors.

Additional evidence implicating $\mathrm{N}$-type calcium channels in anxiety arises from behavioral analysis of novel mouse genetic models, behavioral studies with brain infusions of the $\mathrm{N}$-type calcium channel blocker $\omega$-conotoxin GVIA ( $\omega$-ctx GVIA), and clinical studies in humans. Mice with restricted splice choice in the gene that encodes for the $\alpha_{1}$-pore forming subunit of Cav2.2, Cacna1b (lack 37a-Cacna1b splice variant), show alterations in exploratory behavior in basal conditions and under mild stress [23]. Infusions of $\omega$-ctx GVIA in the medial prefrontal cortex (mPFC) impairs recall of extinguished memories in fear conditioning, and ventricular application of $\omega$-ctx GVIA results in reduced exploratory behavior in the elevated plus maze and light-dark box [24, 25]. However, few studies exist determining the functional expression of $\mathrm{N}$-type calcium channels in areas related to anxiety such as the basolateral amygdala (BLA) and MPFC. 
The BLA and MPFC are well-known areas that encode fear and anxiety in humans and rodents [26]. In rodent models, the BLA and MPFC regulate anxiety- and fear-related behavior in opposing ways [27-30]. Direct activation of the BLA leads to reduced exploratory behavior in the elevated plus maze and enhanced freezing in response to auditory cues initially paired with a shock [31]. By contrast, activation of the MPFC results in enhanced exploratory behavior and reduced freezing in fear conditioning tasks [30]. The BLA and mPFC contain similar cell types. In both areas, several types of interneurons, distinguished by the expression of peptides, have a powerful inhibitory control on PNs. Among the main interneuron types that control PN activity are interneurons that express parvalbumin ( $\left.\mathrm{PV}^{+} \mathrm{INs}\right)$, somatostatin $\left(\mathrm{SOM}^{+} \mathrm{INs}\right)$, vasoactive intestine peptide ( $\left.\mathrm{VIP}^{+} \mathrm{INs}\right)$, and $\mathrm{CCK}^{+} \mathrm{INs}$ $[32,33] . \mathrm{PV}^{+} \mathrm{INs}$ synapse onto cell bodies; $\mathrm{CCK}^{+} \mathrm{IN}$ s synapse onto cell bodies and dendrites; and $\mathrm{SOM}^{+} \mathrm{INs}$ synapse onto dendrites of PNs [34-36]. VIP+INs primarily suppress $\mathrm{SOM}^{+} \mathrm{INs}$, thereby disinhibiting PNs [37]. Previous studies have shown that $\mathrm{CCK}^{+} \mathrm{INs}$ play important roles in fear- and anxiety-related behavior [38]. Selective activation of $\mathrm{CCK}^{+} \mathrm{IN}$ s enhances contextual fear conditioning [39]. $\mathrm{CCK}^{+} \mathrm{INs}$ mediate the effects of endocannabinoids on anxiety and stress [40]. Optogenetic activation of $\mathrm{CCK}^{+} \mathrm{INS}$ of the BLA in vivo facilitates fear extinction [41].

In this body of work, we determined whether N-type calcium channels control GABA release from $\mathrm{CCK}^{+} I \mathrm{Ns}$ in the BLA and mPFC. We used genetic mouse models to specifically label $\mathrm{CCK}^{+} \mathrm{IN}$ s combined with synaptic electrophysiology and pharmacology to record GABA release from $\mathrm{CCK}^{+} \mathrm{IN}$ synapses onto PNs in the BLA and MPFC. We found that $\mathrm{CCK}^{+} \mathrm{INs}$ in the BLA partially rely on N-type calcium channels to release GABA, whereas $\mathrm{CCK}^{+} \mathrm{IN}$ s in the $\mathrm{MPFC}$ lack functional N-type calcium channels. However, N-type calcium channels partially control GABA release from other interneuron types in the mPFC. These observations suggest that $\mathrm{N}$-type calcium channels have a cell- and region-specific role on GABA release in the BLA and $\mathrm{MPFC}$. This is significant because determining the functional expression of $\mathrm{N}$-type calcium channels in these brain areas could strengthen the links between $\mathrm{N}$ type calcium channels and anxiety.

\section{Materials and Methods}

\subsection{Animal Models}

Adult female or male mice were used in all of our experiments. No differences associated with sex were detected in our experiments. To visualize $C C K^{+} I N s$ in the BLA and MPFC, we utilized intersectional labeling with Cre and Flpe recombinases under the CCK and distal-less homeobox 5/6 (DIx5/6) promoters, respectively $[42,43]$. We used this approach because CCK is broadly expressed in both PNs and interneurons, whereas DIx5/6 is restricted to interneurons of the forebrain (Figure 1) $[42,44]$. We first crossed Cck-IRES-Cre mice (The Jackson Laboratory, 012706) with D/x5/6-Flpe mice (The Jackson Laboratory, 010815). The progeny from this initial cross, Cck-IRES-Cre; DIx5/6Flpe (CCK-DIx5/6), were dual transgenic mice with both alleles. Next, we crossed these mice with Ai65(RCFL-tdT-D) mice (The Jackson Laboratory, 021875). Ai65(RCFL-tdT-D) mice express the red fluorescent protein tdTomato (tdT) under the control of two STOP codons. The first STOP cassette is flanked with loxP sites (recognized by $\mathrm{Cre}$ ) and the second is flanked with FRT sites (recognized by Flpe). The progeny from this cross is triple-transgenic mice with the genotype Cck-IRES-Cre; DIx5/6Flpe; Ai65(RCFL-tdT)-D (CCK-Dlx5/6-tdT) (Figure 1A). In these mice, Cre-Lox and Flpe-FRT 
recombination removed the two STOP cassettes, resulting in tdT expression in $\mathrm{CCK}^{+}$INs. Successful labeling of $\mathrm{CCK}^{+} \mathrm{IN}$ s was validated in a previous work from our lab [45].

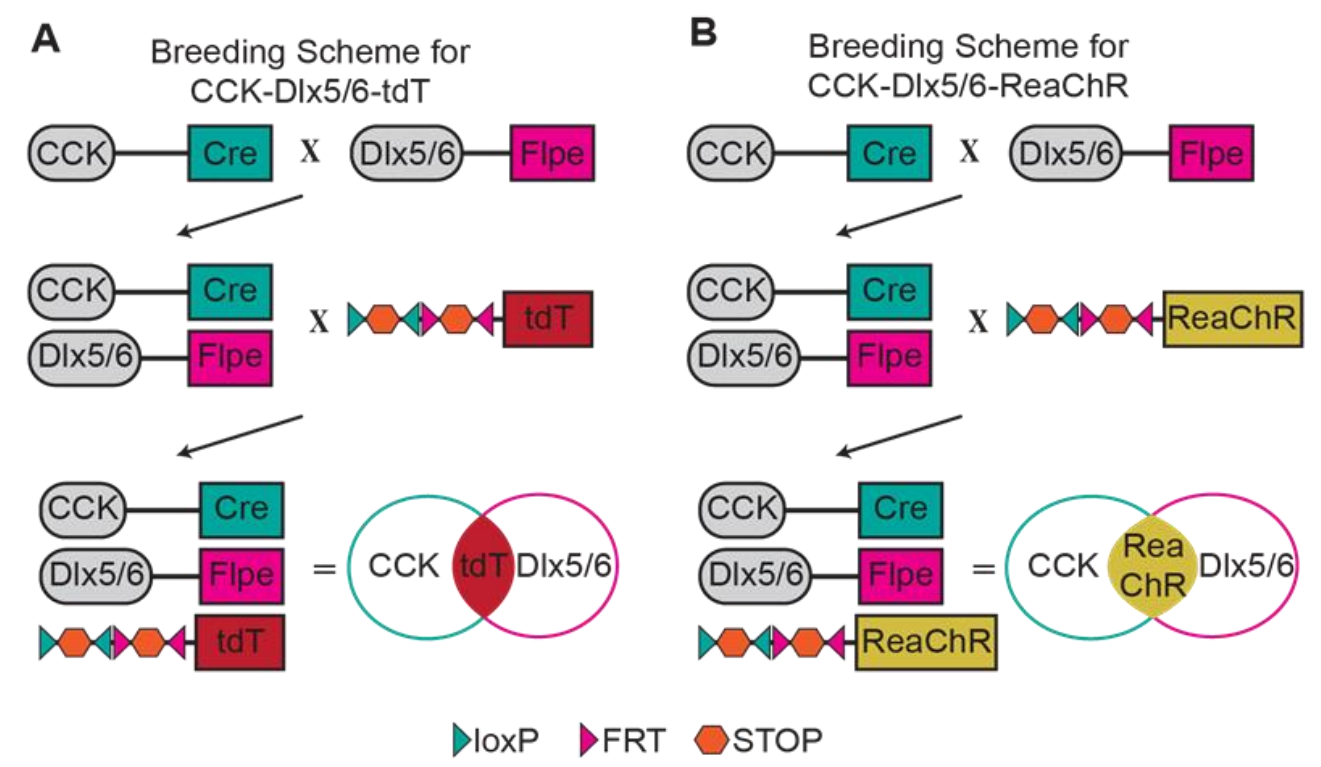

Figure 1 Breeding scheme to generate mice expressing tdT and ReaChR in $\mathrm{CCK}^{+} \mathrm{INs}$ for confocal microscopy and electrophysiology, respectively. A) CCK-Cre mice were bred with Dlx5/6-Flpe mice to generate dual transgenic mice (CCK-D/x5/6), which were crossed with Ai65 mice containing a tdT allele preceded by two STOP codons. Two loxP sites flank the first STOP codon and FRT sites flank the second STOP codon. The offspring of these mice containing the three alleles (CCK-D/x5/6-tdT) resulted in expression of tdT in $\mathrm{CCK}^{+} \mathrm{INs}$. B) To record synapses of $\mathrm{CCK}^{+} \mathrm{INs}$ onto PNs, we generated triple transgenic mice that expresses ReaChR in $C^{2} C^{+} I N s$ and applied optogenetics. Here the dual transgenic mice $C C K-D / \times 5 / 6$ were bred with R26 LSL FSF mice, which contain the allele ReaChR preceded by two STOP codons similar to Ai65 mice. Therefore, after removal of the two STOP codons by $\mathrm{Cre}$ and $F L P e, R e a C h R$ was expressed in $\mathrm{CCK}^{+} \mathrm{INs}$.

To perform electrophysiological studies of synapses between $\mathrm{CCK}^{+} \mathrm{INs}$ and PNs, we used optogenetics and recorded PNs. CCK ${ }^{+}$INs expressed red-shifted channelrhodopsin (ReaChR) fused to $\mathrm{m}$-Citrine. To accomplish this, we generated a second triple transgenic mouse line (Figure 1B). We crossed CCK-DIx5/6 mice to R26 mice (R26 LSL FSF ReaChR-mCitrine, The Jackson Laboratory, 024846), which expressed ReaChR under the control of two STOP codons flanked with loxP and FRT sites. The progeny from this cross are triple-transgenic mice with the genotype Cck-IRES-Cre; DIx5/6Flpe; R26 LSL FSF ReaChR-mCitrine (CCK-D/x5/6-ReaChR).

\subsection{Genotyping}

Mice were genotyped using toe biopsy as done previously [45]. Primer sequences and band size are located in Table 1. 
Table 1 Primer sequences and band sizes for mouse genotyping. Information on the primer sequences and expected PCR products for each mouse line used. F, forward primer sequence; $\mathrm{R}$, reverse primer sequence; $\mathrm{MT}$, mutant; $\mathrm{WT}$, wild-type; $\mathrm{CM}$, common primer; Hom, homozygous; Het, heterozygous; TG, transgene; IC, internal control.

\begin{tabular}{|c|c|c|}
\hline Mouse Line & Primers & Expected Products \\
\hline \multirow{3}{*}{ Cck-IRES-Cre } & F-MT: 5'-TGGTTTGTCCAAACTCATCAA & Hom: 215 bp \\
\hline & F-WT: 5'-GGGAGGCAGATAGGATCACA & Het: 215 and 468 bp \\
\hline & R-CM: 5'-GAGGGGTCGTATGTGTGGTT & WT: 468 bp \\
\hline \multirow{4}{*}{ Dlx5/6-Flpe } & F-TG: 5'-CAGAATTGATCCTGGGGAGCTACG & TG: 406 bp \\
\hline & R-TG: 5'-CCAGGACCTTAGGTGGTGTTTTAC & IC: 200 bp \\
\hline & F-IC: 5'-CAAATGTTGCTTGTCTGGTG & *PCR does not differentiate \\
\hline & R-IC: 5'-GTCAGTCGAGTGCACAGTTT & Hom and Het mice \\
\hline \multirow{4}{*}{$A i 65(R C F L-t d T)-D$} & F-MT: 5'-CTGTTCCTGTACGGCATGG & \\
\hline & R-MT: 5'-GGCATTAAAGCAGCGTATCC & Het. 196 and 297 \\
\hline & F-WT: 5'-AAGGGAGCTGCAGTGGAGTA & WT. 297 \\
\hline & R-WT: 5'-CCGAAAATCTGTGGGAAGTC & \\
\hline \multirow{3}{*}{$\begin{array}{l}\text { R26 LSL FSF ReaChR- } \\
\text { mCitrine }\end{array}$} & R-MT: 5'-CGGGCCATTTAACCGTAAGTTAT & Hom: $284 \mathrm{bp}$ \\
\hline & F-IC: 5'-AAGGGAGCTGCAGTGGAGTA & Het: 284 bp and 297 bp \\
\hline & R-WT: 5'-CCCAAAATCTGTGGGAAGTC & WT: 297 bp \\
\hline
\end{tabular}

\subsection{Histochemistry and Confocal Microscopy}

Adult CCK-D/x5/6-tdT mice were deeply anesthetized with intraperitoneal injections of EUTHASOL ${ }^{\circledR}$ (710101, Virbac Co.). Cardiac perfusions were performed using formalin: PBS solution (HT501128, Sigma-Aldrich). Brains were rapidly dissected and stored at $4{ }^{\circ} \mathrm{C}$ in formalin solution for 24-72 hours for further fixation. $100 \mu \mathrm{m}$ coronal brain slices were prepared using a vibratome (VT1000 S, Leica) and transferred to a 12-well plate (Greiner Bio-One). Slices were washed for ten minutes three times in PBT (0.2\% Triton X, T8787; Sigma-Aldrich and PBS, P3813; Sigma-Aldrich) on an orbital rocker (SK-O180-S, Scilogex). To identify cell bodies, we stained nucleic acids with SYTO13 (S7575, ThermoFisher) by incubating slices for one hour at room temperature in a 1:10000 dilution of SYTO-13 in PBT. Following incubation, slices were washed twice in PBT and once in PBS before mounting. For brain slices with multiple fluorescent proteins, multi-track acquisition was implemented to avoid excitation cross talk. Fluorophores and their spectra were as follows (excitation/emission in $\mathrm{nm}$ ): SYTO-13 (488/509) and tdTomato (554/581). Images were acquired using a Zeiss LSM 510 Meta and the proprietary software.

\subsection{In Vitro Brain Slice Preparation}

Adult CCK-D/x5/6-ReaChR mice were deeply anaesthetized with isoflurane and quickly decapitated. Brains were rapidly removed from the skull and placed into chilled and oxygenated 
$\left(95 \% \mathrm{O}_{2} / \mathrm{CO}_{2}\right)$ artificial cerebral spinal fluid (cutting aCSF) containing high $\mathrm{Mg}^{2+}$ and low $\mathrm{Ca}^{2+}$ (in $\mathrm{mM}$ ): $\mathrm{NaCl}$ (130), $\mathrm{KCl}$ (3.5), $\mathrm{KH}_{2} \mathrm{PO}_{4}(1.1), \mathrm{MgCl}_{2}$ (6), $\mathrm{CaCl}_{2}$ (1), dextrose (10), kynurenic acid (2), $\mathrm{NaHCO}_{3}$ (30), ascorbate (0.4), thiourea (0.8), and sodium pyruvate (2) at pH 7.35 and $310 \mathrm{mOsm} .300 \mu \mathrm{m}$ coronal slices containing the BLA or MPFC were prepared using the vibratome Leica VT1200 S (Leica Biosystems). Slices were transferred from the vibratome to a brain slice chamber (BSKH; Digitimer) containing oxygenated cutting aCSF and kept at $37^{\circ} \mathrm{C}$ for 15 minutes before transferring to a second slice chamber with oxygenated room temperature recording aCSF containing regular $\mathrm{Mg}^{2+}$ and $\mathrm{Ca}^{2+}$ (in $\mathrm{mM}$ ) $\mathrm{NaCl}$ (130), $\mathrm{KCl}$ (3.5), $\mathrm{KH}_{2} \mathrm{PO}_{4}$ (1.1), $\mathrm{MgCl}_{2}$ (1.3), $\mathrm{CaCl}_{2}$ (2.5), dextrose (10), $\mathrm{NaHCO}_{3}$ (30), ascorbate (0.4), thiourea (0.8), and sodium pyruvate (2) at $\mathrm{pH} 7.35$ and $300 \mathrm{mOsm}$. Slices were allowed to stabilize for one hour before transferring to the recording chamber where they remained for no more than two hours during patch-clamp recordings.

\subsection{Electrophysiology}

Whole-cell voltage and current clamp recordings were performed on visually identified PNs of the BLA and mPFC. PNs were identified by their pyramidal-like morphology in the brain slice with differential interference contrast (DIC) microscopy using a camera (01-ROL-BOLT-M-12, QImaging) mounted on an upright microscope (BX51Wl; Olympus). All recordings were performed with a 700B amplifier (Molecular Devices) and digitized with a 1550A analogue/digital convertor (Molecular Devices). Data were acquired at $20 \mathrm{KHz}$ and filtered at $2 \mathrm{KHz}$ with pCLAMP 10 (Molecular Devices). Brain slices were perfused with recording aCSF at $1-2 \mathrm{~mL} / \mathrm{min}$ with constant oxygenation. Borosilicate glass micropipettes with tip resistances between 3-5 $\mathrm{M} \Omega$ were used to perform wholecell current or whole-cell voltage clamp experiments. To record action potentials, we used the following intracellular solution (in mM): K-gluconate (140), HEPES (10), $\mathrm{MgCl}_{2}$ (3), K-ATP (2), $\mathrm{Na}_{2} \mathrm{GTP}$ (0.4), and phosphocreatine (5) at pH 7.4 and 290 mOsm. Action potentials were evoked with square pulses of positive current. To record inhibitory postsynaptic currents (IPSCs), we utilized a cesiumbased intracellular solution consisting of (in $\mathrm{mM}$ ): Cs-gluconate (140), HEPES (10), $\mathrm{MgCl}_{2}$ (3), K-ATP (2), $\mathrm{Na}_{2} \mathrm{GTP}(0.4)$, and phosphocreatine (5) at pH 7.4 and $290 \mathrm{mOsm}$. PNs were patched and held at $-70 \mathrm{mV}$. In optogenetic experiments, IPSCs were evoked with LED light filtered with Texas Red filter. LED stimuli was applied for $0.1 \mathrm{~ms}$ every $10 \mathrm{~s}$. Pulses of red light were controlled with a digital input of a $1550 \mathrm{~A}$ analogue/digital convertor. Series resistance was measured throughout the experiment and cells with an increase in $>10 \%$ of series resistance were discarded. For electrically evoked IPSCs, an electrical current was delivered to brain slices using a current stimulus isolator (A365, WPI) coupled to a tungsten concentric electrode (FHC). Pulses of 0.1 ms were delivered every 10 sec using a digital input of a $1550 \mathrm{~A}$ analogue/digital convertor. Current intensity was adjusted to obtain an IPSC amplitude between 300 and 500 pA, thereby reducing voltage errors.

\subsection{Pharmacology}

Stock concentrations were made at $1000 \mathrm{x}$ working concentrations and stored at $-20^{\circ} \mathrm{C} .100 \mu \mathrm{M}$ BIC (ab120107; Abcam) was added to the bath perfusion to confirm IPSCs. $0.5 \mu \mathrm{M} \omega$-ctx GVIA (ab120215, Abcam) was added to the bath perfusion. $50 \mathrm{mM}$ APV (ab120030; Abcam) and $20 \mathrm{mM}$ NBQX (ab120046, Abcam) was added to the bath to block glutamatergic neurotransmission when electrical stimulation was used. Aliquots were prepared for each stock solution and thawed only once on the day of recordings. 


\section{Results}

We first confirmed that $\mathrm{CCK}^{+} \mathrm{INs}$ are present in the BLA and $\mathrm{mPFC}$ in our CCK-D/x5/6-tdT mice. Immunohistochemistry was performed in sections of both the BLA and MPFC of CCK-D/x5/6-tdT mice. The identity of these cells was previously confirmed using fluorescence activated cell sorting coupled to qRT-PCR. Cells expressing tdT also expressed GABAergic markers such as glutamate decarboxylase-2 mRNA and the cannabinoid receptor 1 mRNA; both are hallmarks of $\mathrm{CCK}^{+} \mathrm{INs}$ [45]. As expected, we found cells labeled with tdT in both areas. $\mathrm{CCK}^{+} \mathrm{INs}$ were scattered throughout the BLA (Figure 2A). Similarly, $\mathrm{CCK}^{+} \mathrm{INs}$ were found distributed across the layers I-VI of the MPFC (Figure 2B). These results suggest that $\mathrm{CCK}^{+} I N$ s in the BLA and $\mathrm{mPFC}$ were successfully labeled using an intersectional genetic labeling approach.
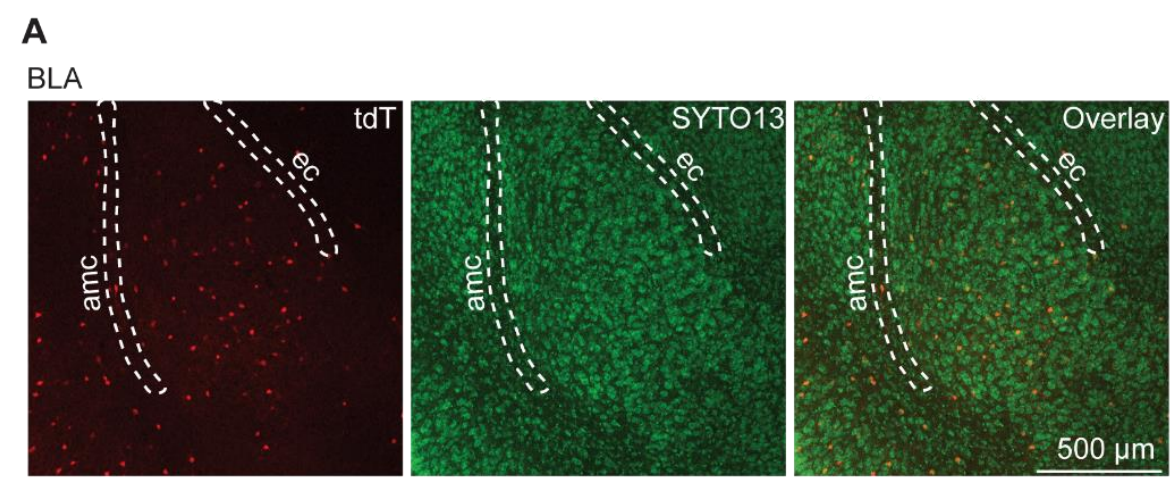

B
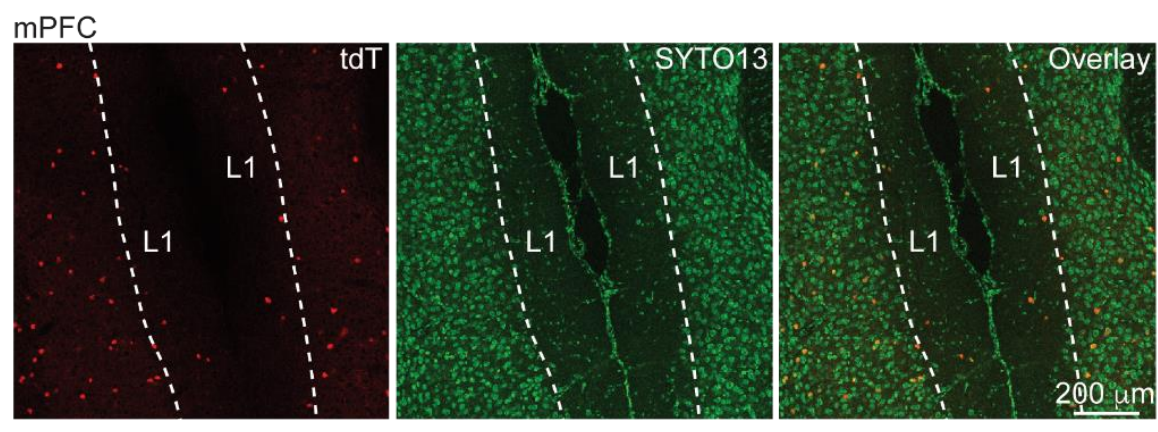

Figure 2 Presence of $\mathrm{CCK}^{+} \mathrm{INs}$ in the BLA and mPFC. A) Representative fluorescence images from the BLA of a CCK-DIx5/6-tdT mouse. $\mathrm{CCK}^{+} \mathrm{INs}$ (red) were found distributed throughout the BLA. Dotted lines describe the amygdalar capsule (amc) and the external capsule (ec). B) Representative fluorescent images from the mPFC of a CCK-D/x5/6-tdT. $\mathrm{CCK}^{+} \mathrm{INs}$ (red) are localized in all layers of the mPFC. Dotted line indicates the limit between layer 1 (L1) and layer 2. SYTO13 (green) was used to stain for cell nuclei in A and $\mathrm{B}$.

Previous results have shown that hippocampal $\mathrm{CCK}^{+} \mathrm{INs}$ rely exclusively on $\mathrm{N}$-type calcium channels to release GABA and immunohistochemistry studies later confirmed that $\mathrm{CCK}^{+}$INs synapses are enriched with $\mathrm{N}$-type calcium channels [12-16]. Therefore, we assessed if $\mathrm{CCK}^{+} \mathrm{INs}$ from the BLA and $\mathrm{mPFC}$ also rely on $\mathrm{N}$-type calcium channels for GABA release. $\mathrm{CCK}^{+} \mathrm{IN}$ s make synaptic contacts with PNs in both the BLA and mPFC, we recorded PNs in CCK-DIx5/6-ReaChR. PNs in the BLA were visually identified using DIC (Figure 3A). The BLA was initially visualized at $4 x$, and PNs were 
identified under 40x based on their pyramidal shape in DIC microscopy (Figure 3A). We confirmed PN identity based on an accommodating firing pattern recorded with current clamp, 85-90\% of recorded cells showed this firing pattern (Figure 3B, left panel). IPSC were recorded using wholecell voltage clamp and optogenetic stimulation. This response was blocked with the GABA blocker bicuculline (BIC), demonstrating that these IPSCs rely only on GABAergic neurotransmission (Figure 3B, right panel). No blockers of excitatory transmission were used in these conditions. Thus, the lack of response after BIC block further supports that stimulating ReaChR results only in GABAergic neurotransmission from $\mathrm{CCK}^{+} \mathrm{INs}$. To determine if $\mathrm{N}$-type calcium channels contribute to GABA release from $\mathrm{CCK}^{+} I N$ s on PNs in the BLA, we applied $\omega$-ctx GVIA to the slice preparation after 20-30 min of stable IPSC recordings. Steady state of IPSC inhibition by $\omega$-ctx GVIA was reached in 20 min (Figure 3C, middle panel). After $\omega$-ctx GVIA block, BIC was applied to confirm that only IPSCs were being recorded (Figure 3C, left and middle panels). We found that $\omega$-ctx GVIA inhibits $\sim 50 \%$ of the IPSC in these conditions (\% $\omega$-ctx GVIA inhibition \pm s.e.m $=50.55 \pm 5.12 \%, n=7$ mice), suggesting that $\mathrm{CCK}^{+} \mathrm{IN}$ synapses on $\mathrm{PNs}$ partially rely on $\mathrm{N}$-type calcium channels.
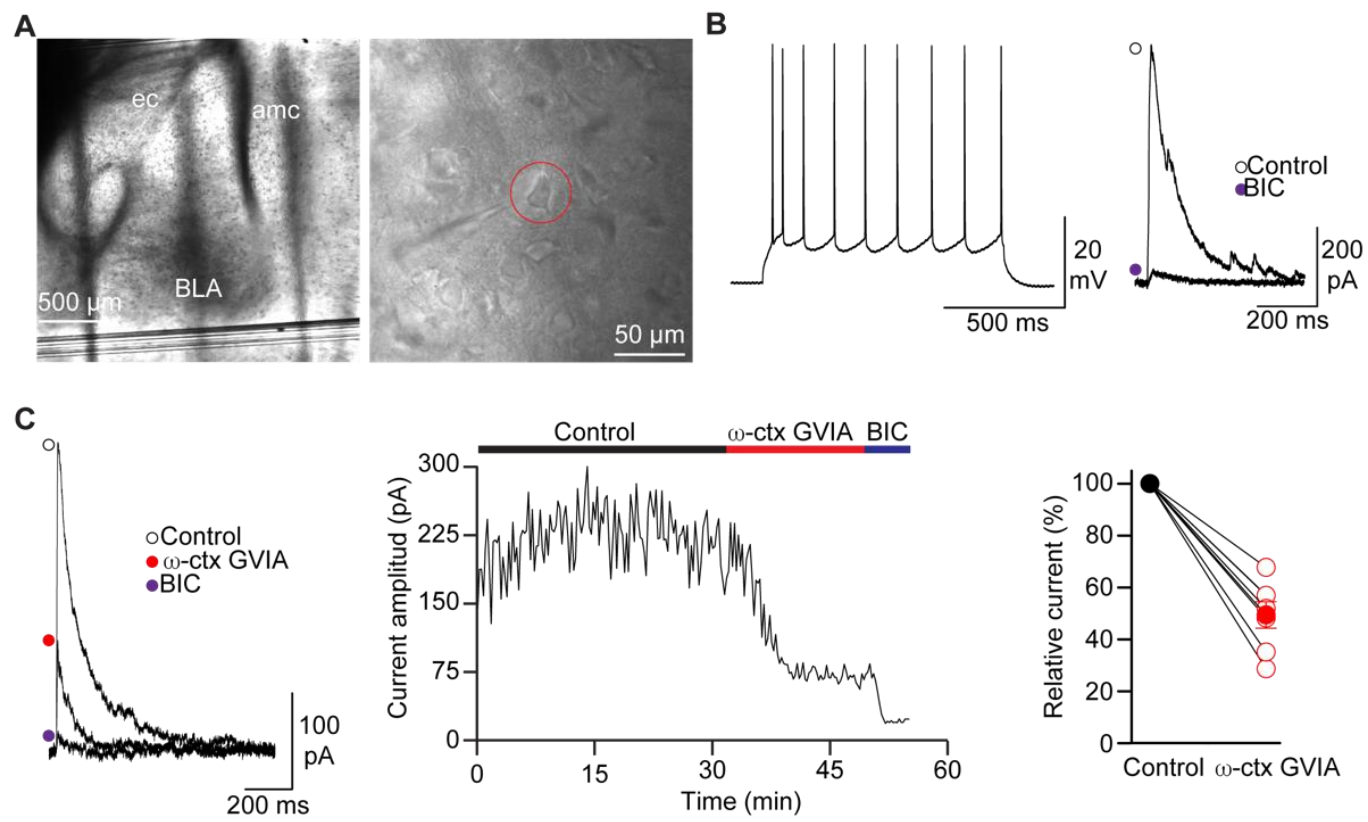

Figure 3 Synapses from $\mathrm{CCK}^{+} \mathrm{INs}$ in BLA partially rely on $\mathrm{N}$-type calcium channels to release GABA. A) Visual identification of PNs in the BLA with DIC microscopy at $4 \mathrm{x}$ (left panel) and 40x (right panel). Note the pyramidal shape of the recorded cell (red circle). ec = external capsule, amc = amygdala capsule. B) Non-accommodating firing pattern characteristic of PNs in the BLA (left panel). Blockage of light-evoked IPSCs with $100 \mu \mathrm{M}$ $\mathrm{BIC}$ in PNs from brain slices of a CCK-DIx5/6-ReaChR mouse (right panel). C) Representative IPSCs with $0.5 \mu \mathrm{M} \omega$-ctx GVIA and $100 \mu \mathrm{M}$ BIC (left panel). Time course of $\omega$-ctx GVIA and BIC block (middle panel). Quantification of IPSC inhibition by $\omega$-ctx GVIA (right panel). IPSC size was determined by fitting current size to a line for $10 \mathrm{~min}$ of stable recordings during control and during $\omega$-ctx GVIA exposure. Percentage of current was calculated relative to the control condition. Filled circles represent average $\%$ of current relative to control and empty circles represent $\%$ of current relative to control for each individual cell. 
$\mathrm{CCK}^{+} \mathrm{INs}$ are also present in the MPFC [43]. We next tested if $\mathrm{CCK}^{+} \mathrm{INs}$ in the mPFC rely on N-type calcium channels to release GABA. Here, we recorded PNs of the mPFC in slices of CCK-D/x5/6ReaChR mice and evoked IPSCs using optogenetics. PNs were identified with DIC. We recorded PNs from layers $2 / 3$ and 5 because they receive abundant synaptic connections from $\mathrm{CCK}^{+}$INs [39]. PNs in layers $2 / 3$ and 5 were visually identified due to their pyramidal shape and the transversal orientation of their dendritic tree (towards layer 1) (Figure 4A). Furthermore, we identified PNs based on their characteristic non-accommodating firing pattern, 85-90\% of recorded PNs showed this firing pattern (Figure 4B, left panel). We recorded IPSCs in PNs, which were confirmed by full BIC block (Figure 4B, right panel). After $15-20$ min of stable IPSC recording, $\omega$-ctx GVIA was perfused and no significant block of the IPSC was observed during 25-30 min of recording after baseline (Figure 4C, left and middle panels). To ensure drugs were being properly perfused into the brain slice, we applied BIC, which induced full block of IPSCs (Figure 4C, middle panel). The lack of $\omega$-ctx GVIA inhibition of IPSCs was observed in $~ 85 \%$ of recorded cells (Figure 4C, right panel), although the other $15 \%$ showed $<10 \%$ inhibition. However, on average, $\omega$-ctx GVIA failed to reduce IPSCs in $\mathrm{CCK}^{+}$IN synapses on PNs of the mPFC (\% $\omega$-ctx GVIA inhibition \pm s.e.m $=3.82 \pm 6.53 \%, n=7$ mice). These results suggest that these synapses rely very little on $\mathrm{N}$-type calcium channels to release GABA, which is in contrast with those results observed in $\mathrm{CCK}^{+} \mathrm{IN}$ synapses of the BLA (comparison of $\% \omega$-ctx GVIA inhibition between the BLA and mPFC, $p=0.0001$, Student's t-test).
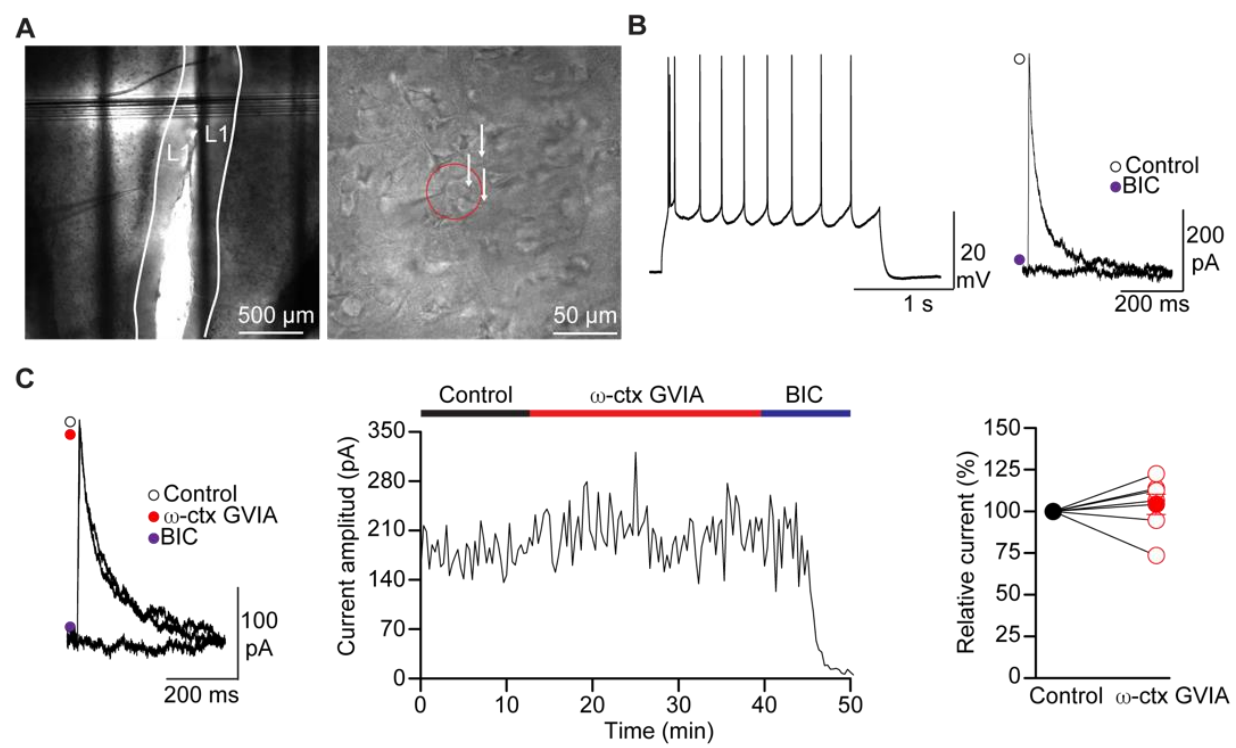

Figure 4 Synapses of $\mathrm{CCK}^{+} \mathrm{INs}$ onto PNs in the MPFC do not rely on $\mathrm{N}$-type calcium channels to release GABA. A) Visual identification of PNs in mPFC layer 1 (L1) with DIC microscopy at $4 x$ (left panel) and 40x (right panel). Note the pyramidal shape of the recorded cell (red circle) and the transversal orientation of dendrites arising from PNs (right panel, white arrows). B) Non-accommodating firing pattern characteristic of PNs in $\mathrm{mPFC}$ (left panel) and pharmacological characterization with $100 \mu \mathrm{M}$ BIC of IPSCs recoded in PNs and evoked with red light in brain slices of CCK-DIx5/6-ReaChR mouse (right panel). C) Representative IPSCs with $0.5 \mu \mathrm{M} \omega$-ctx GVIA and $100 \mu \mathrm{M} \mathrm{BIC} \mathrm{(left}$ panel). Time course of $\omega$-ctx GVIA and BIC block (middle panel). Quantification of IPSC inhibition by $\omega$-ctx GVIA (right panel). Relative current was determined as indicated 
above. Filled circles represent average \% of current relative to control and empty circles represent $\%$ of current relative to control for each individual cell.

We next tested if GABA release from other interneurons relies on $\mathrm{N}$-type calcium channels. We recorded IPSCs in PNs with electrical stimulation using a concentric electrode placed in layer I from WT mice (Figure 5A). PNs were visually identified through their pyramidal shape and transversal dendritic orientation (Figure 5A, right panel). Similarly, we recorded a non-accommodating firing pattern. We confirmed electrically induced IPSCs with block by BIC. In these recordings, excitatory transmission was blocked with APV and NBQX, NMDA and AMPA/Kainate receptor blockers, respectively (Figure $5 B$ ). Full block of the IPSC by BIC in the presence of APV and NBQX suggest that GABA responses were successfully isolated in our experimental conditions. To assess if $\mathrm{N}$-type calcium channels control GABA release, we perfused $\omega$-ctx GVIA after $10-15$ min of stable IPSC recordings, and we observed $\sim 40 \%$ of IPSC reduction $20-30$ min after $\omega$-ctx GVIA perfusion (Figure 5C, middle panel). Inhibition of IPSCs by $\omega$-ctx GVIA was observed in the vast majority of the recorded cells (\% $\omega$-ctx GVIA inhibition \pm s.e. $m=45.72 \pm 8.08 \%, n=6$ mice) (Figure 5C, right panel). Our results show that inhibitory synapses onto PNs rely on $\mathrm{N}$-type calcium channels. Taken together with our previous results, these synapses are likely to belong to non-CCK ${ }^{+}$INs.

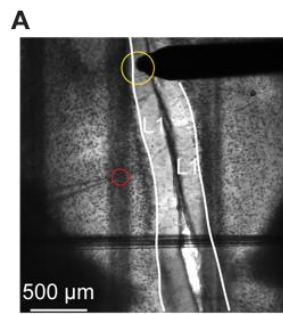

B

C
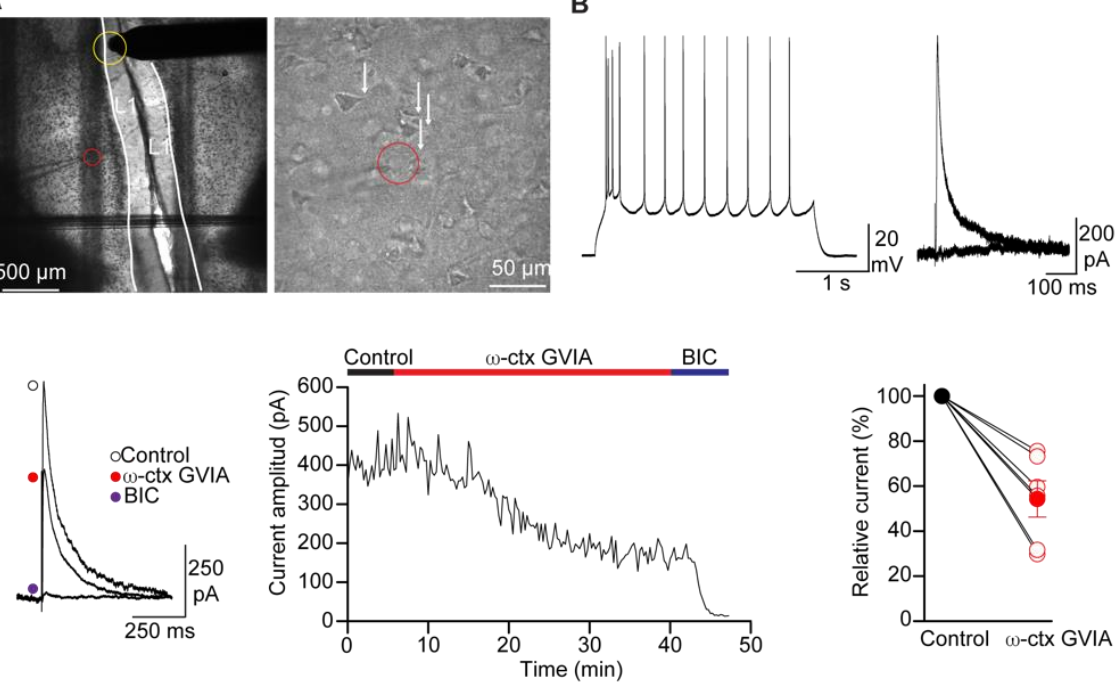

Figure 5 GABAergic neurotransmission in the mPFC partially relies on $\mathrm{N}$-type calcium channels. A) Visual identification of PNs in layer 1 (L1) of the mPFC in WT mice with DIC microscopy at $4 x$ (left panel) and 40x (right panel). Note the pyramidal shape of the recorded cell (red circle) and the transversal orientation of dendrites arising from PNs (right panel, white arrows). Stimulation electrode was placed in L1 as indicated by yellow circle. B) Non-accommodating firing pattern characteristic of PNs from the mPFC (left panel), and pharmacological characterization with $100 \mu \mathrm{M}$ BIC of IPSCs recoded in PNs evoked with electrical stimulation in a WT mouse (right panel). C) Representative IPSCS with $0.5 \mu \mathrm{M} \omega$-ctx GVIA and $100 \mu \mathrm{M}$ BIC (left panel). Time course of $\omega$-ctx GVIA and BIC block (middle panel). Quantification of IPSC inhibition by $\omega$-ctx GVIA (right panel). Percentage of current was determined as indicated previously. Filled circles represent average \% of current relative to control and empty circles represent \% of current relative to control for each individual cell. 


\section{Discussion}

$\mathrm{CCK}^{+}$INs were labeled for histochemistry and electrophysiology using an intersectional labeling approach. Our representative images in figure 2 show that we successfully label CCK+INs using mice with similar genotypes to the ones used in previous studies [35, 39, 42, 43, 45]. We found that IPSCs recorded on $\mathrm{CCK}^{+}$IN/PN synapses in the BLA are partially sensitive to $\omega$-ctx GVIA. This is in contrast with previous observations in synapses of $\mathrm{CCK}^{+} \mathrm{IN}$ s onto CA1 pyramidal cells of the HPC, where $\omega$ ctx GVIA blocks $100 \%$ of the IPSC $[12,16]$. The dominant role of $\mathrm{N}$-type calcium channels on GABA release has been observed in basket $\mathrm{CCK}^{+}$INs and Schaffer collateral associated $\mathrm{CCK}^{+} \mathrm{INs}[13,16]$. In the BLA, $\mathrm{CCK}^{+} \mathrm{IN}$ s are classified into CCK large and small. Large $\mathrm{CCK}^{+} \mathrm{INs}$ synapse onto the cell bodies of PNs that project to the MPFC and they resemble basket CCK INs of the HPC $[31,46]$. BLA small $\mathrm{CCK}^{+}$INs co-express VIP, but their function is not known $[31,46]$. These observations open the possibility that different $\mathrm{CCK}^{+} \mathrm{IN}$ s in the BLA express different proportion of $\mathrm{N}$-type calcium channels. Furthermore, it is also possible that $\mathrm{CCK}^{+} \mathrm{INs}$ express different amounts of calcium channels relative to $\mathrm{CCK}^{+} \mathrm{IN}$ in the HPC. Nonetheless, our results unveil a cell-specific role of $\mathrm{N}$-type calcium channels in controlling transmitter release from $\mathrm{CCK}^{+} \mathrm{IN}$ in the BLA.

In the MPFC, we found that N-type calcium channels play a negligible role in controlling GABA release from $\mathrm{CCK}^{+}$INs compared to the BLA and HPC. This observation further supports a cell- and region-specific role of $\mathrm{N}$-type calcium channels in the control of excitability in areas linked to anxiety. Similar to the BLA, $\mathrm{CCK}^{+} \mathrm{IN}$ s are heterogenous in the cortex; five $\mathrm{CCK}^{+} \mathrm{IN}$ s populations have been identified using single-cell transcriptomic analysis coupled to electrophysiology [47]. This heterogeneity could help explain the differences in the dependence of GABA release on N-type calcium channels between the BLA, MPFC and HPC. Our results align with single-cell transcriptome analysis that shows that the Cacna1b mRNA is expressed at low levels in only one of the five subtypes of cortical $\mathrm{CCK}^{+} \mathrm{IN}$ [47]. However, when using electric stimulation and blocking all of glutamatergic neurotransmission, we found that the IPSCs recorded in PNs of the MPFC were partially sensitive to $\omega$-ctx GVIA. This observation suggests that there are other types of interneurons outside of $\mathrm{CCK}^{+} \mathrm{IN}$ s that utilize $\mathrm{N}$-type calcium channels to release GABA. Accordingly, previous studies have shown that $80 \%$ of the IPSC generated by action potentials in neurogliaform interneurons of rat PFC are blocked with $\omega$-ctx GVIA [48]. But, PV ${ }^{+} I N$ s utilize P/Q-type channels rather than $\mathrm{N}$-type calcium channels [34], which provides further support for the idea that N-type calcium channel are expressed in specific synapses of the mPFC, excluding $\mathrm{CCK}^{+} \mathrm{IN}$ and $\mathrm{PV}^{+} \mathrm{IN}$ synapses. VIP'INs and calbindin-expressing interneurons are also present in the MPFC, however the type of presynaptic calcium channels that these interneurons use to release GABA is unknown.

In our recordings, IPSCs were evoked by shining light on brain slices of mice that express ReaChR2 in $\mathrm{CCK}^{+}$INs. Next, $\omega$-ctx GVIA was added in the same brain slice preparation. The percentage of IPSC inhibition by $\omega$-ctx GVIA was measured as a read out of the presence of N-type channels. The percentage of IPSC inhibition by $\omega$-ctx GVIA was calculated as a ratio of the IPSC amplitude before and after the toxin was added to the preparation. Thus, the percentage of inhibition of IPSC by $\omega$ ctx GVIA is independent of the number of neurons that express ReaChR2. This type of analysis has been performed before by others to demonstrate the role of $\mathrm{N}$-type channels in controlling transmitter release $[12,16]$.

Our results demonstrate that $\mathrm{N}$-type calcium channels partially control GABA release from $\mathrm{CCK}^{+} \mathrm{INs}$ in BLA and other interneurons in $\mathrm{MPFC}$, however, we are unable to rule out whether $\mathrm{P} / \mathrm{Q}$ - 
and/or R-type channels contribute to GABA release from these synapses. These could be part of future studies to fully characterize the type of presynaptic calcium channels that control GABA release from these important interneurons.

Clinical studies have shown that blockage of $\mathrm{N}$-type calcium channels with Ziconotide increase anxiety in humans, interestingly, $\mathrm{CCK}^{+} \mathrm{IN}$ s distribution is conserved between humans, mouse, and rat [49]. Our results open the possibility that blockage of N-type calcium channels in the BLA increases excitability of PNs leading to enhanced anxiety. Previous studies have shown that infusions of $\omega$-ctx GVIA in the infralimbic cortex (ILA) results in failure to recall extinguished fear (a hallmark of anxiety disorders). This observation is in line with an underactive ILA and point to a dominant role of $\mathrm{N}$-type calcium channels in the excitation of PNs via glutamatergic transmission or disinhibition through VIP+INs. Our results support this, given the minor role of N-type calcium channels on GABA release onto PNs of the MPFC.

\section{Conclusions}

In this report, we have successfully labeled $\mathrm{CCK}^{+} \mathrm{INs}$ using an intersectional approach. We recorded synapses of $\mathrm{CCK}^{+} \mathrm{INs}$ onto PNs of the BLA and MPFC. Our results show that the synaptic responses evoked with optogenetics are purely GABAergic. We found that IPSCs from $\mathrm{CCK}^{+}$INs onto PNs of the BLA are sensitive to $\omega$-ctx GVIA but not fully blocked, suggesting that these synapses partially depend on $\mathrm{N}$-type calcium channels to release GABA. We also showed that $\mathrm{CCK}^{+} \mathrm{IN}$ synapses onto PNs of the mPFC are resistant to $\omega$-ctx GVIA, suggesting that these synapses do not rely on $\mathrm{N}$ type calcium channels to release GABA. However, we still found that other GABAergic synapses in the mPFC depend on N-type calcium channels to some degree. Given the prime role of $\mathrm{CCK}^{+} \mathrm{INs}$ in emotional processing, our results shed light on the possible links between $\mathrm{N}$-type calcium channels and anxiety.

\section{Acknowledgments}

We thank Melanie Bertolino and Marie Akiki for their assistance in mouse genotyping.

\section{Author Contributions}

Maxwell Blazon performed the histochemistry and electrophysiological experiments. Brianna LaCarubba and Alexandra Bunda maintained, genotyped and provided input on experimental design. Natalie Czepiel, Shayna Mallat, and Laura Londrigan helped with genotyping and maintenance of the mouse colony. Natalie Czepiel, Shayna Mallat, Laura Londrigan, and Arturo Andrade wrote the manuscript. Arturo Andrade designed the study.

\section{Funding}

This work was supported by the National Institute of Mental Health [grant number, R0OMH099405].

\section{Competing Interests}

Authors declare no competing interests. 


\section{References}

1. Catterall WA. Structure and regulation of voltage-gated $\mathrm{Ca}^{2+}$ channels. Annu Rev Cell Dev Biol. 2000; 16: 521-555.

2. Catterall WA, Perez-Reyes E, Snutch TP, Striessnig J. International Union of Pharmacology. XLVIII. Nomenclature and structure-function relationships of voltage-gated calcium channels. Pharmacol Rev. 2005; 57: 411-425.

3. Schampel A, Kuerten S. Danger: High voltage-the role of voltage-gated calcium channels in central nervous system pathology. Cells. 2017; 6: 43.

4. Zamponi GW, Lewis RJ, Todorovic SM, Arneric SP, Snutch TP. Role of voltage-gated calcium channels in ascending pain pathways. Brain Res Rev. 2009; 60: 84-89.

5. Boland LM, Morrill JA, Bean BP. Omega-conotoxin block of $\mathrm{N}$-type calcium channels in frog and rat sympathetic neurons. J Neurosci. 1994; 14: 5011-5027.

6. Hirning LD, Fox AP, McCleskey EW, Olivera BM, Thayer SA, Miller RJ, et al. Dominant role of Ntype $\mathrm{Ca}^{2+}$ channels in evoked release of norepinephrine from sympathetic neurons. Science. 1988; 239: 57-61.

7. Ino M, Yoshinaga T, Wakamori M, Miyamoto N, Takahashi E, Sonoda J, et al. Functional disorders of the sympathetic nervous system in mice lacking the $\alpha_{1 B}$ subunit $\left(\mathrm{Ca}_{v} 2.2\right)$ of $\mathrm{N}$-type calcium channels. Proc Natl Acad Sci USA. 2001; 98: 5323-5328.

8. Molderings GJ, Likungu J, Göthert M. N-Type calcium channels control sympathetic neurotransmission in human heart atrium. Circulation. 2000; 101: 403-407.

9. Brimblecombe KR, Gracie CJ, Platt NJ, Cragg SJ. Gating of dopamine transmission by calcium and axonal N-, Q-, T- and L-type voltage-gated calcium channels differs between striatal domains. J Physiol. 2015; 593: 929-946.

10. Kato T, Otsu Y, Furune $Y$, Yamamoto T. Different effects of L-, N- and T-type calcium channel blockers on striatal dopamine release measured by microdialysis in freely moving rats. Neurochem Int. 1992; 21: 99-107.

11. Phillips PE, Stamford JA. Differential recruitment of N-, P- and Q-type voltage-operated calcium channels in striatal dopamine release evoked by 'regular' and 'burst' firing. Brain Res. 2000; 884: 139-146.

12. Földy C, Neu A, Jones MV, Soltesz I. Presynaptic, activity-dependent modulation of cannabinoid type 1 receptor-mediated inhibition of GABA release. J Neurosci. 2006; 26: 1465-1469.

13. Hefft $S$, Jonas $P$. Asynchronous GABA release generates long-lasting inhibition at a hippocampal interneuron-principal neuron synapse. Nat Neurosci. 2005; 8: 1319-1328.

14. Lenkey N, Kirizs T, Holderith N, Máté Z, Szabó G, Vizi ES, et al. Tonic endocannabinoid-mediated modulation of GABA release is independent of the CB1 content of axon terminals. Nat Commun. 2015; 6: 6557.

15. Wilson RI, Kunos G, Nicoll RA. Presynaptic specificity of endocannabinoid signaling in the hippocampus. Neuron. 2001; 31: 453-462.

16. Ali $A B$. CB1 modulation of temporally distinct synaptic facilitation among local circuit interneurons mediated by N-type calcium channels in CA1. J Neurophysiol. 2011; 105: 10511062.

17. Scheuber A, Miles R, Poncer JC. Presynaptic Cav2.1 and Cav2.2 differentially influence release dynamics at hippocampal excitatory synapses. J Neurosci. 2004; 24: 10402-10409. 
18. Wu LG, Saggau P. Pharmacological identification of two types of presynaptic voltage-dependent calcium channels at CA3-CA1 synapses of the hippocampus. J Neurosci. 1994; 14: 5613-5622.

19. Wheeler DB, Randall A, Tsien RW. Roles of N-type and Q-type $\mathrm{Ca}^{2+}$ channels in supporting hippocampal synaptic transmission. Science. 1994; 264: 107-111.

20. Saegusa $H$, Kurihara $T$, Zong $S$, Kazuno A, Matsuda $Y$, Nonaka $T$, et al. Suppression of inflammatory and neuropathic pain symptoms in mice lacking the $\mathrm{N}$-type $\mathrm{Ca}^{2+}$ channel. EMBO J. 2001; 20: 2349-2356.

21. Kim C, Jeon D, Kim YH, Lee CJ, Kim H, Shin HS. Deletion of $\mathrm{N}$-type $\mathrm{Ca}\left({ }^{2+}\right)$ channel $\mathrm{Ca}(\mathrm{v}) 2.2$ results in hyperaggressive behaviors in mice. J Biol Chem. 2009; 284: 2738-2745.

22. Nakagawasai $O$, Onogi $H$, Mitazaki S, Sato A, Watanabe $K$, Saito $H$, et al. Behavioral and neurochemical characterization of mice deficient in the $\mathrm{N}$-type $\mathrm{Ca}^{2+}$ channel $\alpha_{1 \mathrm{~B}}$ subunit. Behav Brain Res. 2010; 208: 224-230.

23. Bunda A, LaCarubba B, Bertolino M, Akiki M, Bath K, Lopez-Soto J, et al. Cacna1b alternative splicing impacts excitatory neurotransmission and is linked to behavioral responses to aversive stimuli. Mol Brain. 2019; 12: 81.

24. Zhou Y, Niimi K, Li W, Takahashi E. Cav2. 2-mediated signaling in the neural circuits underlying anxiety. Integr Mol Med. 2015.

25. Zhou Y, Niimi K, Li W, Takahashi E. Inhibition of Cav2. 2-mediated signaling disrupts conditioned fear extinction. Integr Mol Med. 2015.

26. McDonald AJ, Zaric V. GABAergic somatostatin-immunoreactive neurons in the amygdala project to the entorhinal cortex. Neuroscience. 2015; 290: 227-242.

27. Felix-Ortiz AC, Beyeler A, Seo C, Leppla CA, Wildes CP, Tye KM. BLA to vHPC inputs modulate anxiety-related behaviors. Neuron. 2013; 79: 658-664.

28. Kim MJ, Loucks RA, Palmer AL, Brown AC, Solomon KM, Marchante AN, et al. The structural and functional connectivity of the amygdala: From normal emotion to pathological anxiety. Behav Brain Res. 2011; 223: 403-410.

29. Likhtik E, Paz R. Amygdala-prefrontal interactions in (mal)adaptive learning. Trends Neurosci. 2015; 38: 158-166.

30. Quirk GJ, Likhtik E, Pelletier JG, Paré D. Stimulation of medial prefrontal cortex decreases the responsiveness of central amygdala output neurons. J Neurosci. 2003; 23: 8800-8807.

31. Tye KM, Prakash R, Kim SY, Fenno LE, Grosenick L, Zarabi H, et al. Amygdala circuitry mediating reversible and bidirectional control of anxiety. Nature. 2011; 471: 358-362.

32. Capogna M. GABAergic cell type diversity in the basolateral amygdala. Curr Opin Neurobiol. 2014; 26: 110-116.

33. Kawaguchi $Y$, Kondo S. Parvalbumin, somatostatin and cholecystokinin as chemical markers for specific GABAergic interneuron types in the rat frontal cortex. J Neurocytol. 2002; 31: 277-287.

34. Zaitsev AV, Povysheva NV, Lewis DA, Krimer LS. P/Q-type, but not N-type, calcium channels mediate GABA release from fast-spiking interneurons to pyramidal cells in rat prefrontal cortex. J Neurophysiol. 2007; 97: 3567-3573.

35. Nguyen R, Venkatesan S, Binko M, Bang JY, Cajanding JD, Briggs C, et al. Cholecystokininexpressing interneurons of the medial prefrontal cortex mediate working memory retrieval. J Neurosci. 2020; 40: 2314-2331.

36. Tremblay R, Lee $S$, Rudy B. GABAergic interneurons in the neocortex: From cellular properties to circuits. Neuron. 2016; 91: 260-292. 
37. Pi HJ, Hangya B, Kvitsiani D, Sanders JI, Huang ZJ, Kepecs A. Cortical interneurons that specialize in disinhibitory control. Nature. 2013; 503: 521-524.

38. Babaev O, Piletti Chatain C, Krueger-Burg D. Inhibition in the amygdala anxiety circuitry. Exp Mol Med. 2018; 50: 18.

39. Whissell PD, Bang JY, Khan I, Xie YF, Parfitt GM, Grenon M, et al. Selective activation of cholecystokinin-expressing GABA (CCK-GABA) neurons enhances memory and cognition. eNeuro. 2019; 6: ENEURO.0360-18.2019.

40. Lutz B, Marsicano G, Maldonado R, Hillard CJ. The endocannabinoid system in guarding against fear, anxiety and stress. Nat Rev Neurosci. 2015; 16: 705-718.

41. Rovira-Esteban L, Gunduz-Cinar O, Bukalo O, Limoges A, Brockway E, Müller K, et al. Excitation of diverse classes of cholecystokinin interneurons in the basal amygdala facilitates fear extinction. eNeuro. 2019; 6: ENEURO.0220-19.2019.

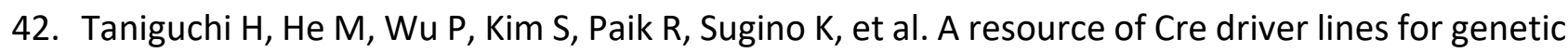
targeting of GABAergic neurons in cerebral cortex. Neuron. 2011; 71: 995-1013.

43. Whissell PD, Cajanding JD, Fogel N, Kim JC. Comparative density of CCK- and PV-GABA cells within the cortex and hippocampus. Front Neuroanat. 2015; 9: 124.

44. Batista-Brito R, Machold R, Klein C, Fishell G. Gene expression in cortical interneuron precursors is prescient of their mature function. Cereb Cortex. 2008; 18: 2306-2317.

45. Bunda A, LaCarubba B, Akiki M, Andrade A. Tissue- and cell-specific expression of a splice variant in the II-III cytoplasmic loop of Cacna1b. FEBS Open Bio. 2019; 9: 1603-1616.

46. Vogel E, Krabbe S, Gründemann J, Cusulin JI, Lüthi A. Projection-specific dynamic regulation of inhibition in amygdala micro-circuits. Neuron. 2016; 91: 644-651.

47. Fuzik J, Zeisel A, Máté Z, Calvigioni D, Yanagawa Y, Szabó G, et al. Integration of electrophysiological recordings with single-cell RNA-seq data identifies neuronal subtypes. Nat Biotechnol. 2016; 34: 175-183.

48. Dalby NO, Grunnet M. Voltage gated N-type Ca-channels in neurogliaform interneurons in the rat prefrontal cortex. bioRxiv. 2018.

49. Katona I, Sperlágh B, Maglóczky Z, Sántha E, Köfalvi A, Czirják S, et al. GABAergic interneurons are the targets of cannabinoid actions in the human hippocampus. Neuroscience. 2000; 100 : 797-804.

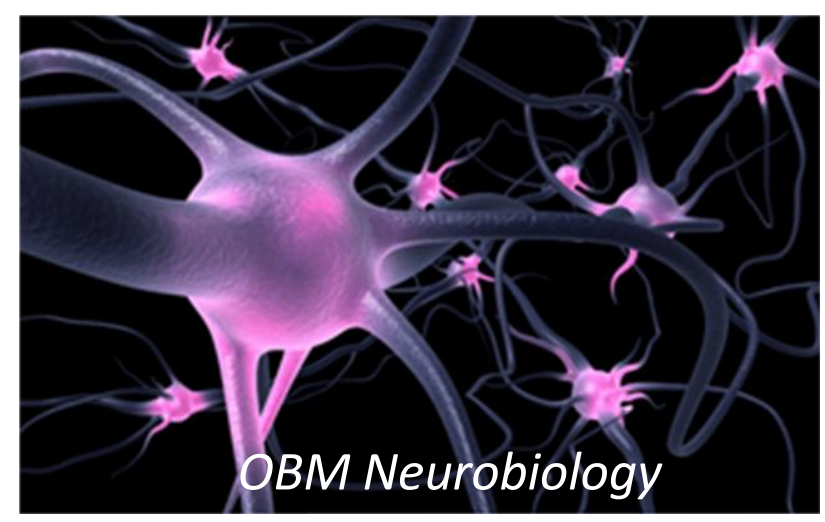

Enjoy OBM Neurobiology by:

1. Submitting a manuscript

2. Joining volunteer reviewer bank

3. Joining Editorial Board

4. Guest editing a special issue

For more details, please visit:

http://www.lidsen.com/journals/neurobiology 